\title{
Title: Structural Determinants of LTA4H Aminopeptidase Activation
}

Authors: Schroeder M. Noble ${ }^{1}$, Kyung Hyeon Lee ${ }^{1}$, Elaine Cagnina ${ }^{2}$, Hoyoung Lee ${ }^{2}$, Marie Burdick $^{2}$, Y. Michael Shim ${ }^{2}$ and Mikell Paige ${ }^{3}$

${ }^{1}$ Department of Wound Infections, Bacterial Diseases Branch, Walter Reed Army

Institute of Research, 503 Robert Grant Avenue, Silver Spring, Maryland, USA, 20910.

${ }^{2}$ University of Virginia, Department of Medicine, Division of Pulmonary and

Critical Care Medicine. Charlottesville, VA

${ }^{3}$ Department of Chemistry and Biochemistry, George Mason University, Manassas, VA

Leukotriene A4 hydrolase ( $\left.\mathrm{LTA}_{4} \mathrm{H}\right)$ possesses opposing functions by activating both proinflammatory and anti-inflammatory pathways. In pro-inflammatory pathways the epoxy hydrolase (EH) activity of LTA 4 H catalyzes hydrolysis of leukotriene $\mathrm{A}_{4}\left(\mathrm{LTA}_{4}\right)$ to $\mathrm{LTB}_{4}$, a pro-inflammatory lipid mediator that contributes to diseases such as adult respiratory distress syndrome (ARDS), irritable bowel syndrome (IBS), and chronic obstructive pulmonary disease (COPD). In anti-inflammatory pathways, the aminopeptidase (AP) activity of $\mathrm{LTA}_{4} \mathrm{H}$ catalyzes the hydrolysis of the peptide proline-glycine-proline (PGP), a chemotactic peptide reported to be associated with neutrophilic inflammation. The disease pathology associated with $\mathrm{LTA}_{4} \mathrm{H}$ makes this enzyme an attractive target for therapeutic intervention. Several groups have targeted $\mathrm{LTA}_{4} \mathrm{H}$ for development of therapeutics by non-selectively targeting $\mathrm{LTA}_{4} \mathrm{H} \mathrm{EH}$ function. However, many of these inhibitors have failed to show clinical benefit. Our therapeutic strategy is to develop anti-inflammatory compounds that selectively potentiate $\mathrm{LTA}_{4} \mathrm{H}$ AP activity, while preserving EH activity. Previously, we evaluated enhancement of $\mathrm{LTA}_{4} \mathrm{H}$ AP activity in kinetics assays with the small molecule 4MDM, tested 4MDM in two murine in vivo models, and determined the crystal structure of $\mathrm{LTA}_{4} \mathrm{H}$ in complex with 4MDM. In this effort, we have synthesized new LTA ${ }_{4} \mathrm{H}$ AP activators, evaluated the compounds kinetically and determined the $2.8 \AA$ crystal structure of $\mathrm{LTA}_{4} \mathrm{H}$ in complex with one of these activators. The new activator was bound in each of the 3 molecules of LTA $4 \mathrm{H}$ in the asymmetric unit. The 4MDM portion of the new activator bound in the same orientation as in the $\mathrm{LTA}_{4} \mathrm{H}: 4 \mathrm{MDM}$ complex structure. The presence of a heterocyclic group appended to 4MDM shifted the 4MDM group $\sim 1.0 \AA$ towards the $\mathrm{LTA}_{4} \mathrm{H}$ AP active site. The methoxy group interacted with the main-chain carbonyl of Q136 and limits rotational freedom of this residue. The constraints on this residue offers insight into the differential effects of the 4MDM analog on AP activity as compared to 4MDM or ARM1. In conclusion, we have demonstrated potentiation of $\mathrm{LTA}_{4} \mathrm{H}$ AP activity by new anti-inflammatory compounds in vitro, and determined the crystal structure of $\mathrm{LTA}_{4} \mathrm{H}$ bound to one of these compounds. This structure will aid in the next round of design and synthesis of $\mathrm{LTA}_{4} \mathrm{H}$ AP activators. 